\title{
A chemsex crucible: the context and the controversy
}

\section{David Stuart}

Substance Use Lead, GUM/HIV, 56 Dean Street, London, UK

\section{Correspondence to} Mr David Stuart, GUM/HIV, 56 Dean Street, Chelsea and Westminster Hospital NHS Foundation Trust, London W1D 6AQ, UK; david.stuart@me.com

\section{CrossMark}

To cite: Stuart D. J Fam Plann Reprod Health Care 2016;42:295-296.

\section{INTRODUCTION}

The public health response to chemsex, when understood objectively, is actually a simple model of multidisciplinary interventions. Just as opiate addiction requires behavioural therapies, medicine, community engagement, and an understanding of the motivations for use, chemsex requires - well, exactly the same. Many excellent models of chemsex support already exist internationally. ${ }^{1-3}$

Unfortunately, however, you can't remove the sex from chemsex; and where there is sex, there are moral and religious judgments and stigmas. Make that gay sex, include HIV, and chemsex becomes something that can require some untangling before an effective public health response can be mounted.

\section{DEFINING CHEMSEX}

The untangling must, therefore, begin with a definition. Chemsex is a word invented on geo-sexual networking apps by gay men (and adopted by the gay men's health sector) that defines a syndemic of specific behaviours associated with specific recreational drugs, and is particular to a specific, high-risk population.

Though the media spotlight may have distorted the term to define the use of any drugs in sexual contexts by any population, ${ }^{4}$ chemsex actually refers to the use of any combination of drugs that includes crystal methamphetamine, mephedrone and/or gammahydroxybutyrate $(\mathrm{GHB}) /$ gammabutyrolactone (GBL), used before or during sex by men who have sex with men (MSM). These drugs provide users with a particular sexual disinhibition, appetite and duration that set them apart from previously favoured recreational drugs (such as ecstasy, cocaine, marijuana and alcohol), which never manifested the amount of sexual-related harm/infections, injecting harms, deaths, addictions or lifestyle deterioration as these modern-day drugs have amongst modern gay communities.

Chemsex is less of a traditional sex and drug issue than it is an issue related to the use of changing technologies, easy availability of certain recreational drugs via these technologies, and adopted by a small, international but very sexually active cohort of men who are considerably more likely to contract HIV through this behaviour than their non-MSM counterparts (due to high prevalence within that population and the higher number of partners that can be facilitated by the drugs and apps).

Chemsex is also defined by its association with some cultural idiosyncrasies unique to gay cultures that may complicate sex for gay men. These can include early experiences of gay sex and 'coming out' issues, sexualised gay scenes and media, definitions of gay sexual liberation, societal and internalised homophobia, and concepts of risk, fear and stigma born of a 30-year HIV epidemic. It is important that the health sector remains vigilant in providing care for anyone using drugs or alcohol for sex, but also that a particular focus is given to MSM using these drugs in this context as this represents a particular challenge to public health and communities, and some redesigning of the way in which support services function. It is this unique set of harms and motivations that led clinicians to call for chemsex to be considered a public health priority in $2015 .^{5}$

\section{THE CHEMSEX PREVALENCE QUESTION}

No emerging trend or epidemic can be adequately addressed if the prevalence can't be quantified. And therein lies the source of a great deal of the controversy surrounding chemsex. It is still an emerging phenomenon as far as the medical community is concerned, with a specific and complex definition that requires an 
intimate understanding of the drugs' characteristics and effects, and how they differ from other recreational drugs used in the same contexts. This challenges our research on this subject, and the question as to how many people are engaging in chemsex remains unanswerable, at least to date. Some frontline sexual health clinics are capturing some data, ${ }^{6}$ but it is still early days, and these data may lack context if they are attracting the highest-risk cohorts. Population-level surveys, conducted online or at gay Pride events for example, can also lack context if drug users - whose use is often associated with fear of disclosure, or denial - might be reluctant to participate or to disclose honestly.

So the prevalence question remains: are all gay men doing chemsex? Of course not, and no one thinks that. It is, however, important to remember that there is a generation of gay men who remember the stigmatising AIDS headlines from the 1980s, many of whom worked tirelessly to undo the stigma caused to gay communities by those headlines, and fought defiantly to define gay sexual liberation during those harrowing years. Many of these men would understandably be very fearful that chemsex headlines might set the movement back to 1985 , be very resentful of news stories on the subject, and resent any evidence that chemsex may be more prevalent than they'd prefer. It may even manifest as chemsex denialism. It has been a harrowing few decades for a certain generation, and we must be sympathetic and mindful of this fact.

\section{CONCLUSIONS}

Personally, I've been pleased to observe an admirable and objective response from the healthcare community. I've never met anyone who assumed that all gay men do chemsex, or that all drug use manifests problematically. It has mostly been identified as a simple healthcare issue affecting a small but significant and particularly vulnerable population of men. It is an issue that requires further research and an immediate skilling up of frontline staff in sexual health settings. It is also something that requires a robust and kind dialogue within gay communities; ${ }^{7}$ a dialogue that bravely explores and seeks to understand how this unique set of behaviours came to affect so many among us, and devastatingly so, in too many cases. This exploration may unravel some unpleasant truths; it may require some reinvention of gay 'scenes', some redefining of what gay sexual liberation means to a new generation of gay men in 2016; a generation facing new challenges, stigmas, technologies, and a very different relationship with HIV.

So despite the controversy, chemsex is brilliantly simple. We need to research and respond to the healthcare needs of a vulnerable population engaging in chemsex, and to begin a kind, community-led conversation about the underlying motivations. We have endured worse, through the HIV/AIDS epidemic, and if we ignore the tempest within the crucible, we can lick this too.

Twitter Follow David Stuart at @davidastuart

\section{REFERENCES}

1 Victorian AIDS Council (VAC). Sex, drugs and HIV/STIs. http://www.vac.org.au/sex-drugs-and-hivstis [accessed 11 August 2016].

2 La Fuente Hollywood Treatment Center. La Fuente LGBT Gay drug rehab LosAngeles. http://lafuentehollywood.com/ gay-drug-rehab-los-angeles/ [accessed 11 August 2016].

356 Dean Street. Chemsex support at 56 Dean Street. http:// dean.st/chemsex-support/ [accessed 11 August 2016].

4 Knapton S. Chemsex: the alarming new trend of 72 hour drug-fuelled sex sessions. The Telegraph, 3 November 2015. http://www.telegraph.co.uk/news/health/news/11972817/ Chemsex-the-alarming-new-trend-of-72-hour-drug-fuelled-sexsessions.html [accessed 11 August 2016].

5 McCall H, Adams N, Mason D, et al. What is chemsex and why does it matter? BMJ 2015;351:h5790.

6 Stuart D, Nwokolo N, McOwan A, et al. ChemSex: data on recreational drug use and sexual behaviour in men who have sex with men (MSM) from a busy sexual health clinic in London, UK. Conference report, 15 th European AIDS Conference (EACS 2015), 21-24 October 2015.

7 Let's Talk About Gay Sex and Drugs. YouTube video. https:// www.youtube.com/playlist?list=PL59WY5a9gIGQ2DQJMYp dyDQzvBFO7zIw [accessed 11 August2016].

\section{In Memory of Egon Diczfalusy}

Egon Diczfalusy, the father of the fetal endocrinology discipline, died on 19 September 2016, aged 96 years. Diczfalusy gained international fame for his work on the definition of the human feto-placental unit and thereafter the main focus of his scientific work were the hormones involved in human reproduction. He oversaw the World Health Organization's 'Expanded Programme of Research, Development and Research training in Human Reproduction' for 25 years, helping establish international networks of researchers dedicated to the development of fertility-regulating agents and combating infertility across the world. Diczfalusy leaves as his legacy the Egon and Ann Diczfalusy Foundation, created to support science and development in Eastern Europe. 